\section{Getting Involved in MRS}

Among the many pleasures of being the President of the Materials Research Society are all the people I get to meet. Many of them already work in volunteer roles for the Society, and a significant number of others have asked me how they might get involved. MRS is a member-run and member-driven society and there is always a need for members to volunteer to help run it; so here is a quick tutorial in the opportunities that MRS offers.

Broadly speaking, there are three kinds of volunteer involvement. First, and most prominent, member volunteers organize the technical programs of our meetings and the technical content of MRS Bulletin. Perhaps less prominent, but equally important, members serve on a number of standing committees that run the Society. Finally, members may be elected to the Society's governing board (the Council) or as an officer of the organization.

Most begin their involvement by coorganizing a symposium at a Spring or Fall meeting. A typical symposium has three or four organizers, who are selected by the Meeting Chairs. (The Meeting Chairs are, themselves, selected by the MRS Vice President, mostly from the ranks of experienced symposium organizers.) Although symposium topics might be repeated on various cycles, we do not allow the same group of symposium organizers to repeat the role, so we have a constant need for new symposium organizers. To be a symposium organizer, you need to plan to work on a meeting at least a year-and-a-half in the future. You can identify the appropriate Meeting Chairs on the MRS Web site (www.mrs.org), and then start networking. If you want to organize a symposium in an ongoing series, or on a subject related to a recent symposium, you should discuss your desire with the previous group of symposium organizers to see if they know of anyone else also planning to propose this project. If you want to organize a symposium in a new area, you may need to suggest your own group of symposium organizers-bearing in mind that the Meeting Chairs will have the last say on whether the symposium is included in their meeting, and who organizes it. As a general rule, to be a symposium organizer, you will need to be well-connected in the subject on which you want to work, but you do not necessarily need to have a high level of seniority. It helps to be well organized, too. The ideal group of symposium organizers is balanced with respect to the

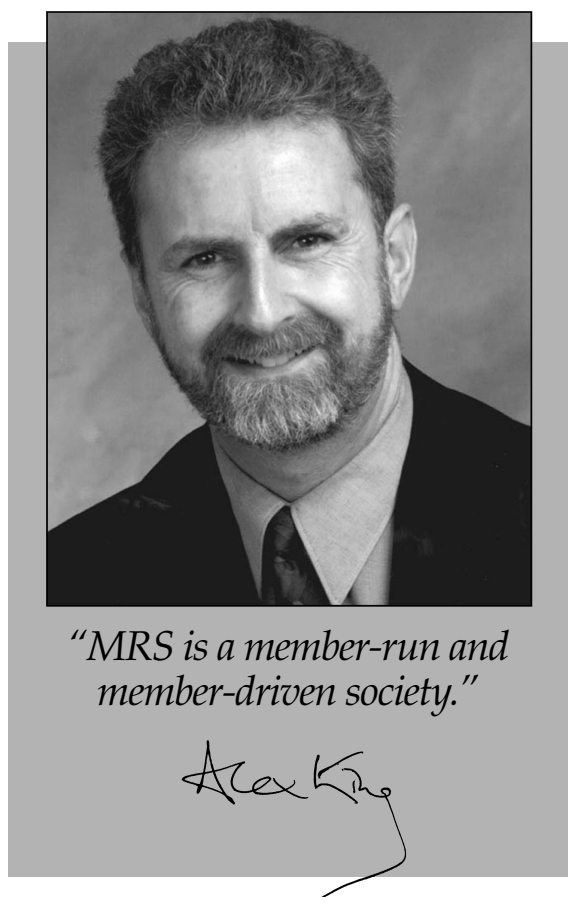

seniority of its members; whether they work in a university, national laboratory, or industry; whether they work within or outside the United States; and whether they are male or female. They also need to cover as many subfields as possible within their symposium topic. Most importantly, though, you need to be in touch with the Meeting Chairs about 18 months before the meeting that you want to help organize.

Topical focus issues of MRS Bulletin, and the corresponding guest editors, are selected in a very similar manner to the symposia and their organizers at the meetings, except that the role taken by the Meeting Chairs is taken by the Volume Organizers for the Bulletin. If you want to offer to organize the articles for an issue of the Bulletin, your networking goals are the same as if you wanted to be a symposium organizer, but your target is the group of Volume Organizers.

If you want to volunteer for a standing committee, the process is very different. The standing committees are increasingly empowered to act on behalf of the Society within the areas of their mandates. They are able to do things and make things happen in a wide variety of ways, depending largely upon the energy and leadership of their members. A complete list of the MRS standing committees can be found on the MRS Web site, and I urge you to take a look to see if there are any committees that you would like to join. Appointments to standing committees are made by the President, usually after discussion with the committee chair, so it is a good idea to talk to the chair of any committee that you would like to join, then contact me (or other presidents in succeeding years). We like to maintain the same balance in our standing committees that we try to achieve in our groups of symposium organizers. If you want to serve the Society in this role, you should routinely plan to attend at least one (or preferably both) of the MRS technical meetings each year, since this is where the committees meet. Many of them also hold telephone conference calls, or other "in-person" meetings in addition to the San Francisco (Spring) and Boston (Fall) meetings, since there is usually some work to be done between meetings, too.

Service on the governing body of the Society calls for election by the entire membership, but the slate of candidates is selected by the Nominating Committee, which is chaired by the Past President. If you want to run for Council or as an officer, contact the Past President very early in the calendar year. Candidates for election typically have some experience in one of the roles just described, so I encourage you to start there.

The efforts of MRS member volunteers are guided and supported by the MRS headquarters, and one of the constant refrains from volunteers is the praise they sing for the staff. The staff culture is one of supporting and effectuating the work of the member volunteers and this makes it possible for your time to be spent with maximal efficiency.

Across all areas of the Society, we always need new volunteers; especially those based in industry, those based outside the United States, and those from traditionally under-represented groups. You will start by networking, and your networking skills will be developed to a high level through volunteer involvement in MRS. The best place to network, of course, is at the Spring and Fall meetings, and the best way to start is by presenting your work as a talk or a poster, and also as a publication in the Proceedings. Get involved: It's your Society. It may occasionally be hard work, but the rewards will be great, and you can expect to have a significant effect on the directions that we take.

AlEx KING 2002 MRS President alexking@ecn.purdue.edu 